Research Article

\title{
Formulation and Pharmaceutical Assessment of Annona muricata Oral Capsules and Suspension as Antidiarrhea Dosage Forms
}

\author{
Frederick William Akuffo Owusu $\left(\mathbb{D},{ }^{1}\right.$ Mariam El Boakye-Gyasi ${ }^{D},{ }^{1}$ Philomena Entsie $\left(\mathbb{D},{ }^{2}\right.$ \\ Christina Osei-Asare $\mathbb{D},{ }^{3}$ Ofosua Adi-Dako $\left(\mathbb{D},{ }^{4}\right.$ Grace Boakye Bosuthe, ${ }^{3}$ \\ Esther Antwi-Mensah, ${ }^{3}$ and Marcel Tunkumgnen Bayor ${ }^{1}{ }^{1}$
}

\author{
${ }^{1}$ Department of Pharmaceutics, Faculty of Pharmacy and Pharmaceutical Sciences, \\ Kwame Nkrumah University of Science and Technology, Kumasi, Ghana \\ ${ }^{2}$ Department of Herbal Medicine, Faculty of Pharmacy and Pharmaceutical Sciences, \\ Kwame Nkrumah University of Science and Technology, Kumasi, Ghana \\ ${ }^{3}$ Department of Pharmaceutics, School of Pharmacy, Central University, Miotso, Ghana \\ ${ }^{4}$ Department of Pharmaceutics and Microbiology, School of Pharmacy, University of Ghana, Accra, Ghana
}

Correspondence should be addressed to Frederick William Akuffo Owusu; frederickakuffo.owusu@knust.edu.gh

Received 18 October 2021; Revised 15 November 2021; Accepted 3 December 2021; Published 14 December 2021

Academic Editor: Vinod Kumar Tiwari

Copyright (C) 2021 Frederick William Akuffo Owusu et al. This is an open access article distributed under the Creative Commons Attribution License, which permits unrestricted use, distribution, and reproduction in any medium, provided the original work is properly cited.

\begin{abstract}
Annona muricata (Annonaceae) is a tropical plant widely known for its edible fruits. Recent scientific studies have confirmed the folkloric use of its seeds as an antidiarrheal agent. This study sought to formulate capsules and suspensions using the ethanolic extract from Annona muricata seeds. The dried ethanolic extract was formulated into granules and subsequently encapsulated. The suspension formulated was assessed for sedimentation rate, sedimentation volume, viscosity, dissolution, drug content, and flow rate, while pharmacopeia tests such as disintegration, dissolution, uniformity of weight, and drug content were carried out on the formulated capsules. The formulated suspension passed the drug content and in vitro release studies. Annona muricata suspension exhibited pseudoplastic flow with good sedimentation rate and sedimentation volume. The formulated capsules passed the in vitro dissolution studies, weight uniformity, disintegration, and drug content tests. The ethanolic extract of Annona muricata seeds was appropriately formulated into standardized solid and liquid oral dosage forms.
\end{abstract}

\section{Introduction}

Diarrhea is a condition of having unusual loose or watery stools with a sense of urgency. It is also known to occur when the lining of the intestine is unable to retain fluid, leading to lose stool. The precarious nature of the disease is linked with dehydration due to fluid loss and consistently high morbidity in the absence of vigorous interventions. Diarrhea is a leading cause of death in all age groups with an estimated 1.31 million deaths recorded annually across the globe for a mortality rate of $7.58 \%[1,2]$. The burden of deaths is greatest in low-income countries such as Ghana where getting access to portable water and proper sanitation is a problem. In Ghana, the mortality rate as at 2020 was $7.15 \%$ per 1000 inhabitants with diarrhea accounting for about $25 \%$ of death in children under five years with correspondingly high debilitating effects in adults [1-4].

Most patients with diarrhea do not seek medical attention unless symptoms last for more than three days or they have coexisting fever, prostration, or rectal bleeding by which time their condition may have worsened. Hence, it is very imperative that medications used in the treatment of diarrhea are effective and readily available $[1,3]$. In order to treat diarrhea, various orthodox medicines have been 
developed. However, the World Health Organization promotes studies on medicinal plants that can be used to manage and treat diarrhea and to formulate these medicinal plant extracts in standardized dosage forms that will be convenient, efficient, and safe for the patient. In Ghana, traditionally, a lot of plants are used to treat diarrhea including Bridelia ferruginea, Albizia ferruginea, and Psidium guajava (guava). Another plant commonly used to treat diarrhea in Ghana is Annona muricata [5-7].

Annona muricata of the family Annonaceae, popularly known as soursop, graviola, or guanabana is a fruit that grows in the tropical regions of America, Africa, and the Caribbean. Phytochemical studies carried out on various parts of the plant revealed the presence of tannins, phenolic compounds, flavonoids, and alkaloids which underpin their pharmacological relevance $[6,8]$. Further research carried out by Doe et al. shows that the ethanolic extract of the seeds have antidiarrheal property and corroborates its folkloric use [9]. Irrespective of the reported dose and pharmacological activity of the ethanolic extract of the seeds of Annona muricata in the management of diarrhea, its use is limited in clinical settings due to the absence of standardized dosage forms. Against this background, this study seeks to provide standardized dosage forms (suspensions and capsules) of the ethanolic extract of the seeds of Annona muricata in order to substantiate its clinical usefulness and integration into current health care delivery as an effective antidiarrheal agent.

\section{Materials and Methods}

2.1. Materials. Annona muricata fruits (Ashaiman, Ghana), maize starch (Anhui Sunhere Pharmaceuticals, China), acacia and tragacanth powder (Sigma Aldrich), benzoic acid, and laboratory grades of concentrated hydrochloric acid and ethanol (Department of Pharmaceutics, KNUST, Ghana) were obtained.

\subsection{Methods}

2.2.1. Collection of Sample and Extractive Procedure. Annona muricata fruits were obtained from the Ashaiman market, in the Greater Accra region of Ghana, and authenticated at the Department of Pharmacognosy, KNUST, Kumasi, Ghana. Extraction was done using a previously established method [9]. The seeds were removed from the fruits. It was then dried under shade for 7 days, and the weight was taken afterwards. The dried seeds were milled into fine powder and weighed as well. The milled seeds were then macerated in $80 \%$ ethanol in an airtight container and stored in the refrigerator at $5^{\circ} \mathrm{C}$ for 7 days. The decanted solution was filtered using a filter paper, and the filtrate was evaporated using a rotary evaporator and then freeze dried into fine powder.

2.2.2. Assessment of the Flow Characteristics. The indirect method described by Aulton and Taylor [10] in the characterization of the flow properties of materials was used.
2.3. Formulation of Annona muricata Suspension. In the formulation of $100 \mathrm{~mL}$ suspension, Annona muricata extract was weighed and added to the previously weighed Acacia and triturated with benzoic acid and double strength chloroform water to obtain a uniform mixture (such that each $5 \mathrm{~mL}$ contains $0.15 \mathrm{~g}$ ). Water was then used to top up to the mark (Table 1). The excipients were selected after several preformulation studies with different excipients.

2.4. Formulation of Annona muricata Granules. In preparing the granules, starch, lactose, and Annona muricata extract were weighed and mixed using geometric dilution (Table 2). The powdered mixture was then blended with tragacanth mucilage to form a damp mass and prepared into granules (14.8 g) using the method described by [10]. The excipients were selected after several preformulation studies with different excipients.

2.5. Evaluation of Flow Properties of Annona muricata Granules. The flow properties of the formulated granules were determined using the fixed height method for angle of repose and bulk density measurements (Hausner ratio and Carr's index) [10].

2.6. Encapsulation of Annona muricata Granules. The granules were encapsulated using capsule size 1 with $0.15 \mathrm{~g}$ of extract in each capsule (150 mg per dose). Fifty-five (55) capsules were filled simultaneously.

2.7. Quality Assessment of Formulated Capsules. The weight uniformity and disintegration tests were determined as described by the British and International Pharmacopeias $[11,12]$.

2.7.1. Drug Content. Ten capsules were used for this experiment. A capsule was emptied, and $20 \mathrm{~mL}$ of $0.1 \mathrm{M} \mathrm{HCl}$ was added. The mixture was filtered, and $0.1 \mathrm{M} \mathrm{HCl}$ was used to top it up to $50 \mathrm{ml}$. Drawell's UV spectrometer was used in measuring the absorbances at $300 \mathrm{~nm}$ after serially diluting the solutions. The recorded absorbance was then inserted into a calibration equation, and the percentage content was determined for each capsule [11, 13-15].

2.7.2. In Vitro Dissolution Test. In vitro dissolution study was done using the USP type I basket dissolution apparatus. $900 \mathrm{~mL}$ of $0.1 \mathrm{M} \mathrm{HCl}$ was transferred into each of the six dissolution vessels, the temperature of the dissolution vessel was maintained at $37 \pm 2^{\circ} \mathrm{C}$, and a randomly selected capsule was dropped into the dissolution medium. An aliquot of the sample was withdrawn at regular time intervals $(5,15,30,35$, 40,45 , and 60 minutes), and the same volume of $0.1 \mathrm{M} \mathrm{HCl}$ was used to replace the volume withdrawn. The samples were filtered and analyzed with the UV spectrometer at $300 \mathrm{~nm}$. The absorbances obtained were inserted into the calibration equation to obtain the amount of drug released at each time point. A graph of cumulative drug released against time was then plotted to obtain the dissolution profile of the formulated capsules in $0.1 \mathrm{M} \mathrm{HCl}[11,13-15]$. 
TABLE 1: Formula for preparing Annona muricata suspension.

\begin{tabular}{lc}
\hline Ingredient & Quantity \\
\hline Annona muricata extract & $3 \mathrm{~g}$ \\
Acacia powder & $3 \mathrm{~g}$ \\
Benzoic acid & $0.1 \mathrm{~g}$ \\
Double strength chloroform water & $50 \mathrm{~mL}$ \\
Water & $100 \mathrm{~mL}$ \\
\hline
\end{tabular}

TABle 2: Formula for preparing Annona muricata capsules.

\begin{tabular}{lc}
\hline Ingredient & Quantity $(\mathrm{g})$ \\
\hline Annona muricata extract & 10 \\
Starch & 3.2 \\
Lactose & 0.5 \\
Tragacanth mucilage $(10 \% \mathrm{w} / \mathrm{v})$ & 0.7 \\
\hline
\end{tabular}

2.8. Quality Assessment of Annona muricata Suspension. The appearance, $\mathrm{pH}$, rheology, apparent viscosity, drug content, in vitro dissolution studies, sedimentation volume, and sedimentation rate were determined using previously described methods [11, 16-18].

2.8.1. Uniformity in Drug Content of Formulated Annona muricata Suspension. Five (5) $\mathrm{mL}$ of the formulated suspension was diluted with $25 \mathrm{~mL}$ of $0.1 \mathrm{M} \mathrm{HCl}$, filtered, and topped up to $50 \mathrm{~mL}$ using the same solvent $(0.1 \mathrm{M} \mathrm{HCl})$. Drawell's UV spectrometer was used in measuring the absorbances at $300 \mathrm{~nm}$. The recorded absorbance was then inserted into the calibration equation, and the percentage content was determined. This was repeated for nine other $5 \mathrm{~mL}$ aliquots [11, 16-18].

2.8.2. In Vitro Dissolution Study of Annona muricata Suspension. The USP type II paddle dissolution apparatus was used with $900 \mathrm{~mL}$ of $0.1 \mathrm{M} \mathrm{HCl}$ (as the dissolution medium) heated to $37^{\circ} \pm 2^{\circ} \mathrm{C}$. The paddles were set to a speed of $50 \mathrm{rpm}$, and $5 \mathrm{ml}$ of the prepared suspension was transferred into the dissolution medium. After 5, 15, 30, 35, 40,45 , and 60 minutes, an amount of $10 \mathrm{~mL}$ of the dissolution medium was withdrawn, filtered, and the absorbance at $300 \mathrm{~nm}$ was determined using Drawell's UV spectrometer. The withdrawn volume was replaced with same volume of $0.1 \mathrm{M} \mathrm{HCl}$ to maintain sink conditions. The absorbances obtained were inserted into the previously determined calibration equation to obtain the amount of drug released at each time point. A graph of cumulative drug released against time was then plotted to obtain the dissolution profile of the formulated suspension in $0.1 \mathrm{M} \mathrm{HCl}[11,16-18]$.

\section{Results and Discussion}

3.1. Flow Characteristics of Granules and Extract. The powdered extract showed good flow properties with Hausner's ratios of 1.12, Carr's index of $10.98 \%$, and angle of repose of $20.5^{\circ}$, while the Annona muricata granules exhibited excellent flow properties with Hausner's ratios of 1.10 , Carr's index of $8.93 \%$, and angle of repose of $17.5^{\circ}$. This confirms that the formulated granules will improve the encapsulation process by enhancing the uniform filling of the capsule shells $[11,13]$.

\subsection{Assessment of Annona muricata Capsules}

3.2.1. Capsule Weight Uniformity. The weight uniformity of Annona muricata capsules as given in Table 3 complied with the weight uniformity specifications according to the International Pharmacopeia [12]. This implies that each manufactured capsule potentially has the exact amount of the Annona muricata extract and excipients specified, with little variability, reducing the risk of under dosing and overdosing.

3.2.2. Disintegration Profile of Formulated Capsules. Based on the results given in Table 4, the formulated capsules passed the disintegration test as stipulated by the British Pharmacopeia [11]. This indicates that the capsules will release their contents within time for further dissolution of the extract to occur.

3.2.3. Drug Content. The percentage drug content of all ten sampled capsules were within the standard range of 85.0-115\% (Table 5) and confirms that the encapsulation process as well as other preencapsulation procedures were carried out accurately. The results also indicate that the formulated capsules will provide the required therapeutic dose without any overdose or underdosage that would be harmful or detrimental to the patient.

3.2.4. In Vitro Dissolution of Annona muricata Extract from Capsules. According to the data (Figure 1), 99.29\% of the extract was released at the 45 th minute. This implies that the capsules passed the dissolution test and can dissolve well in physiological solution, allowing the extract to be absorbed and the intended pharmacological activity to be achieved $[11,13]$.

\subsection{Quality Assessment of Annona muricata Suspension}

3.3.1. Appearance Test. Over the course of four weeks, the formulated suspension was closely monitored for the appearance or absence of undesired physical changes. There were no unwanted physical changes in the prepared suspension (Table 6). This shows that the suspension is physically stable and retains its aesthetic attractiveness and that the excipients employed in the formulation were appropriate $[10,19]$.

3.3.2. Sedimentation Volume and Rate of Formulated Suspension. A good suspension, in order to improve uniform and accurate dosing, should not have the suspended particles settling quickly. The sedimentation volume of an ideal suspension should be close to 1 with a correspondingly low sedimentation rate [19-21]. This property was evident in the formulated suspension (Figure 2). 
Table 3: Weight uniformity of formulated capsules.

\begin{tabular}{lccc}
\hline Quantity of capsules used & Average weight $(\mathrm{g})$ & Quantity of capsules deviating by $\pm 5 \%$ & Quantity of capsules deviating by $\pm 10 \%$ \\
\hline 20 & 0.450 & None & None \\
\hline
\end{tabular}

Table 4: Disintegration time of formulated capsules.

\begin{tabular}{lccc}
\hline Disintegration test & $1^{\text {st }}$ determination & $2^{\text {nd }}$ determination & Average \\
\hline Time (minutes) & 2.60 & 2.40 & $2.50 \pm 0.14$ \\
\hline
\end{tabular}

TABLE 5: Assay of formulated capsules.

\begin{tabular}{lr}
\hline Capsule number & Drug content (\%) \\
\hline 1 & $99.32 \pm 0.02$ \\
2 & $91.86 \pm 0.03$ \\
3 & $101.53 \pm 0.10$ \\
4 & $95.59 \pm 0.08$ \\
5 & $99.32 \pm 0.06$ \\
6 & $95.59 \pm 0.05$ \\
7 & $101.53 \pm 0.04$ \\
8 & $91.86 \pm 0.06$ \\
9 & $101.53 \pm 0.07$ \\
10 & $99.32 \pm 0.04$ \\
\hline
\end{tabular}

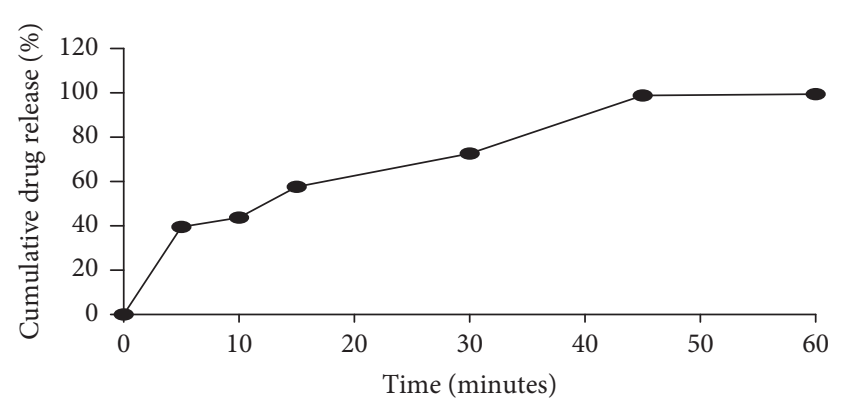

Figure 1: Release profile of Annona muricata extract from capsules.

TABLE 6: Physical stability of formulated suspension.

\begin{tabular}{ll}
\hline Week & Physical instability (aggregation, caking, crystal growth formation) \\
\hline 1 & No physical instability observed \\
2 & No physical instability observed \\
3 & No physical instability observed \\
4 & No physical instability observed \\
\hline
\end{tabular}

3.3.3. Rheology, Redispersibility, and $p H$ of Formulated Suspension. There was no statistical variation $(P \geq 0.05)$ in the $\mathrm{pH}$ of the formulated suspension (Table 7 ) to indicate any physicochemical change $[19,22,23]$. The flow time of the suspension was short with a correspondingly high apparent viscosity for easy pourability (Table 7) $[16,19,22]$. Throughout the study period, the solid particles were redispersed with less than ten (10) strokes (Table 7). The prepared suspension possessed pseudoplastic flow, which implies that it may be swiftly redispersed with modest agitation, and the appropriate amount can be easily drawn (Figure 3). This feature is critical for a good suspension [24].
3.3.4. Drug Content. All sampled doses passed the drug content test (Table 8) [11]. This implies that each volume will have the exact amount of extract needed, preventing overdosing and underdosing, both of which are hazardous to the patient's health.

\subsubsection{In Vitro Dissolution Profile of Annona muricata Extract} from Formulated Suspension. According to the data (Figure 4), $99.20 \%$ of the extract was released at the 45 th minute which complies with pharmacopeia specifications. This implies that the capsules can dissolve well in physiological solution, allowing the extract to be absorbed and eliciting the intended therapeutic effect $[11,22]$. 


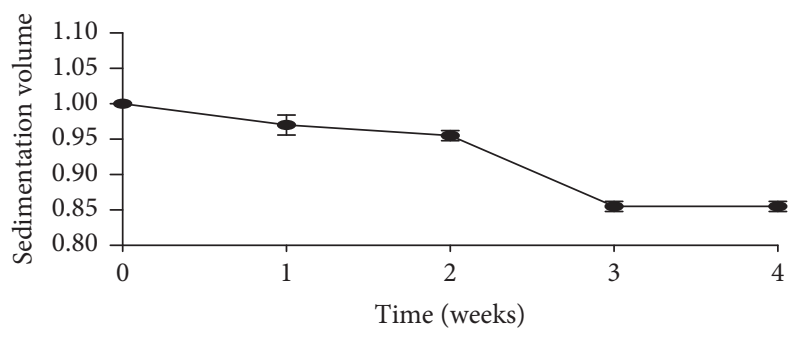

FigURE 2: Effect of time on the sedimentation volume of Annona muricata suspension.

TABle 7: Physicochemical properties of Annona muricata suspension.

\begin{tabular}{lcccc}
\hline Week & $\mathrm{pH}$ & Flow time $(\mathrm{s})$ & Apparent viscosity $(\eta)(\mathrm{mL} / \mathrm{s})$ & Redispersibility (number of strokes) \\
\hline 0 & $6.93 \pm 0.012^{\text {ns }}$ & $8.68 \pm 0.58$ & $1.153 \pm 0.28$ & 7 \\
1 & $6.94 \pm 0.013^{\text {ns }}$ & $8.67 \pm 0.03$ & $1.155 \pm 0.19$ & 7 \\
2 & $6.92 \pm 0.010^{\text {ns }}$ & $8.73 \pm 0.50$ & $1.147 \pm 0.31$ & 9 \\
3 & $6.93 \pm 0.016^{\text {ns }}$ & $8.64 \pm 0.45$ & $1.159 \pm 0.37$ & 7 \\
4 & $6.93 \pm 0.014^{\text {ns }}$ & $8.62 \pm 0.25$ & $1.161 \pm 0.09$ & 7 \\
\hline
\end{tabular}

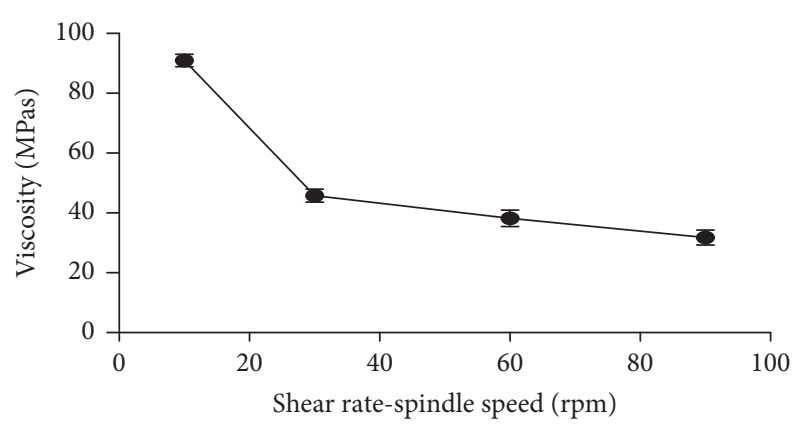

FIGURE 3: Rheology of formulated suspension.

TABle 8: Assay of formulated suspension.

\begin{tabular}{lcc}
\hline Sampled dose number & Absorbance & Drug content $(\%)$ \\
\hline 1 & 0.43 & 91.86 \\
2 & 0.43 & 91.86 \\
3 & 0.46 & 101.53 \\
4 & 0.44 & 95.59 \\
5 & 0.43 & 91.86 \\
6 & 0.45 & 99.32 \\
7 & 0.46 & 101.53 \\
8 & 0.46 & 101.53 \\
9 & 0.45 & 99.32 \\
10 & 0.44 & 95.59 \\
\hline
\end{tabular}

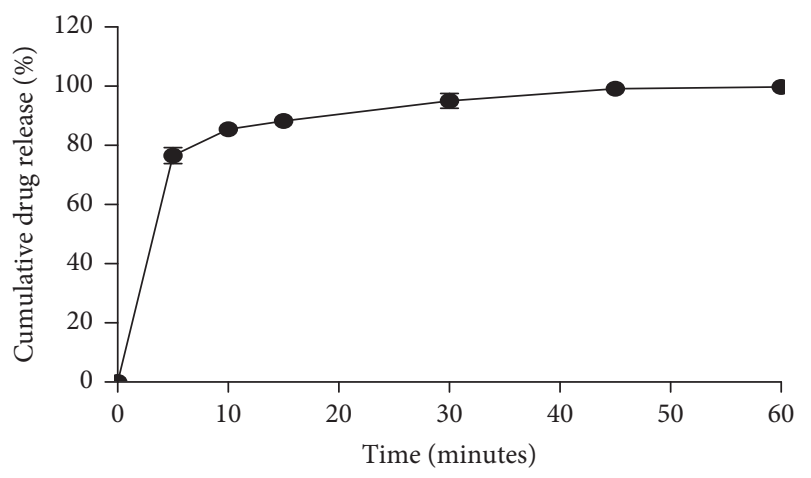

FIGURE 4: Release profile of Annona muricata extract from suspension. 


\section{Conclusion}

This study has shown that the ethanolic extract of Annona muricata can be successfully formulated into capsules and suspension. The prepared dosage forms satisfied the pharmacopeia requirements for quality evaluation and may be used as appropriate alternatives in the management and treatment of diarrhea.

\section{Data Availability}

The data used to support the findings of this study are included within the article and also available from the corresponding author upon request.

\section{Conflicts of Interest}

The authors declare that there are no conflicts of interest.

\section{Acknowledgments}

The authors would like to express their gratitude to the technical personnel of the Department of Pharmaceutics at KNUST.

\section{References}

[1] M. E. Anna, T. B. John, M. A. Penny et al., "Diarrhea in children less than two years of age with known HIV status in Kisumu, Kenya," International Journal of Infectious Diseases, vol. 14, no. 3, pp. 220-225, 2010.

[2] World Health Organization, The Integrated Global Action Plan for the Prevention and Control of Pneumonia and Diarrhea (GAPPD) Ending Preventable Child from Pneumonia and Diarrhea by 2025, WHO, Geneva, Switzerland, 2013.

[3] J. Tetteh, W. K. Takramah, M. A. Ayanore, A. Adoliba Ayanore, E. Bisung, and J. Alamu, "Trends for diarrhea morbidity in the Jasikan district of Ghana: estimates from district level diarrhea surveillance data, 2012-2016," Journal of Tropical Medicine, vol. 2018, Article ID 4863607, 10 pages, 2018.

[4] UNICEF, One Is Too Many: Ending Child Deaths from Pneumonia and Diarrhoea, UNICEF, New York, NY, USA, 2016.

[5] U. Usunomena and O. N. Paulinus, "Phytochemical analysis and mineral composition of Annona muricata leaves," International Journal for Researcher Development, vol. 1, no. 1, pp. 38-42, 2015.

[6] A. V. Coria-Téllez, E. Montalvo-Gónzalez, E. M. Yahia, and E. N. Obledo-Vázquez, "Annona muricata: a comprehensive review on its traditional medicinal uses, phytochemicals, pharmacological activities, mechanisms of action and toxicity," Arabian Journal of Chemistry, vol. 11, no. 5, pp. 662-691, 2018.

[7] J. W. Ahs, W. Tao, J. Löfgren, and B. C. Forsberg, "Diarrheal diseases in low-and middle-income countries: incidence, prevention and management," The Open Infectious Diseases Journal, vol. 4, no. 1, pp. 67-76, 2010.

[8] E. B. Bento, E. F. F. Matias, F. E. Brito et al., "Association between food and drugs: antimicrobial and synergistic activity of Annona muricata L," International Journal of Food Properties, vol. 16, no. 4, pp. 738-744, 2013.
[9] P. Doe, A. Iddrisu, A. Iddrisu et al., "Evaluation of the antidiarrheal activity of the ethanolic seed extract of Annona muricata," The Journal of Phytopharmacology, vol. 8, no. 4, pp. 199-202, 2019.

[10] M. E. Aulton and K. M. Taylor, Aulton's Pharmaceutics E-Book: The Design and Manufacture of Medicines, Elsevier Health Sciences, Amsterdam, Netherlands, 2017.

[11] B.R.I.T.I.S.H. and British Pharmacopoeia Commission, Pharmacopoeia, The Stationery Office, London, UK, 2018.

[12] World Health Organization, International Pharmacopoeia, World Health Organization, Geneva, Switzerland, 9th edition, 2019.

[13] D. Kumadoh, J. Adotey, K. Ofori-Kwakye, S. L. Kipo, T. Prah, and S. Patterson, "Development of oral capsules from Enterica herbal decoction-a traditional remedy for typhoid fever in Ghana," Journal of Applied Pharmaceutical Science, vol. 5, no. 4 , pp. 83-88, 2015.

[14] D. Jyothi, M. Koland, S. Priya, and J. P. James, "Formulation of herbal capsule containing Trigonella foenum-graecum seed extract for the treatment of diabetes," Journal of Young Pharmacists, vol. 9, no. 3, pp. 352-356, 2017.

[15] J. Raphael, M. Bayor, and J. Adotey, "Formulation and evaluation of Bridelia ferruginea and Canthium glabriflorum herbal capsules," Journal of Current Research in Ayurvedic and Pharmaceutical Sciences, vol. 1, no. 7, pp. 11-18, 2011.

[16] E. E. Oppong, C. H. Osei-Asare, and M. W. Klu, "Evaluation of the suspending properties of shea tree gum," International Journal of Pharmacy and Pharmaceutical Sciences, vol. 8, no. 7, pp. 409-413, 2016.

[17] H. S. Mahmud, A. R. Oyi, T. S. Allagh, and M. S. Gwarzo, "Evaluation of the suspending property of Khaya snegalensis gum in co-trimoxazole suspensions," Research Journal of Applied Sciences, Engineering and Technology, vol. 2, no. 1, pp. 50-55, 2010.

[18] F. W. Owusu, C. O. Asare, P. Enstie et al., "Formulation and in vitro evaluation of oral capsules and suspension from the ethanolic extract of cola nitida seeds for the treatment of diarrhea," BioMed Research International, vol. 2021, Article ID 6630449, 7 pages, 2021.

[19] M. Uddin, A. Mamun, N. Akter, M. Sarwar, M. Rashid, and M. Amran, "Pharmacopoeial standards and specifications for pharmaceutical oral liquid preparations," Archives of Current Research International, vol. 3, no. 2, pp. 1-12, 2016.

[20] G. S. Banker and C. T. Rhodes, Modern Pharmaceutics, Marcel Dekker, New York, NY, USA, 4th edition, 2000.

[21] S. P. Agarwal and R. Khanna, Physical Pharmacy, CBS Publishers \& Distributors, New Delhi, India, 2nd edition, 2006.

[22] R. S. Kumar and T. N. S. Yagnesh, "Pharmaceutical suspensions: patient compliance oral dosage forms," World Journal of Pharmacy and Pharmaceutical Sciences, vol. 7, no. 12, pp. 1471-1537, 2016.

[23] S. Bajaj, D. Singla, and N. Sakhuja, "Stability testing of pharmaceutical products," Journal of Applied Pharmarceutical Science, vol. 2, no. 3, pp. 129-138, 2012.

[24] S. Mohanan and A. Krishna, "Formulation and evaluation of liquid oral suspension of paracetamol using newly isolated and characterized hygrophila spinosa seed mucilage as suspending agent," Asian Journal of Pharmaceutical and Clinical Research Research, vol. 11, no. 11, pp. 437-441, 2018. 